\title{
Optimization of the photothermal camera for crack detection
}

\author{
by J.-C. Krapez ${ }^{1}$, L. Legrandjacques ${ }^{2}$, F. Lepoutre ${ }^{1}$ and D. L. Balageas ${ }^{1}$ \\ ${ }^{1}$ ONERA, DMSE, BP 72, F-92322 CHÂTILLON-Cedex, France \\ ${ }^{2}$ FRAMATOME, Technical Centre, BP13, F-71380 SAINT MARCEL, France. email: krapez@onera.fr
}

\begin{abstract}
We present recent advances about the application of the photothermal camera for the detection of cracks on metallic surfaces of industrial quality. We describe a normalization procedure designed to clean the photothermal images of the optical noise and thereby to increase the propability of detection of the cracks. A theoretical analysis about the spatial resolution of the photothermal camera is finally exposed.
\end{abstract}

\section{Introduction}

The detection of cracks is particularly important for safety in aircraft and nuclear industries. Today's industrial methods however are rather slow. Visual testing methods are still the most widely used especially during the processing steps and the maintenance operations. Among them, penetrant testing is probably the most popular one because its cost is low and its versatility large. Nevertheless, in some cases, this simple technique cannot be used: unsafe human interventions, forbidden contact with the surface specimen, high risk of false alarms (generally associated with surface roughness), necessity of on-line or automated controls...

A few years ago, several teams in the world have imagined a new kind of camera, called flying spot or photothermal camera, able to solve the problem of the crack detection [1-7]. In this system, the surface heating is achieved by the absorption of a CW laser which scans the surface. Focalisation of the heating beam allows for a three dimensional heat diffusion sensitive to defects perpendicular to the surface (other localized heat sources can also be used, see e.g. [7]). The infrared emission is monitored by an infrared sensor, the image of which scans with an adjustable offset the surface at the same velocity as the laser (Fig. 1).

\section{Thermal signature of cracks in the absence of optical artefacts}

The presence of a transverse component for heat flux leads to the detection of surface breaking cracks through the thermal barrier effect induced by that crack: when the excitation/detection system approaches a crack, the IR signal first increases since the nominal heat diffusion is hampered in the direction of the crack. When the detector passes over the crack, the signal suddenly decreases below the nominal level since the detector now senses the temperature of a region which received less energy than a sound region. The signal finally reaches its standard value again when the spots are far from the crack. This bipolar shape of the detected temperature from part to part of the standard temperature is called the thermal signature of the crack (see fig. 4).

A continuous monitoring of the IR signal along the scanning and the reconstruction of this information in a 2-D representation leads to an image of the test piece where the surface defects are made visible.

\section{Retrieval of the thermal signature of cracks in the presence of optical artefacts}

On surfaces of industrial interest, the thermal signature of the crack is most often hidden by the large variations of the local emissivity $\varepsilon(x)$ and absorptivity $\alpha(x)$ produced by the crack. For instance, on a polished metal the product $\varepsilon(x) \alpha(x)$ varies from $10^{-2}$ to 1 if the crack is 
considered as a black body, while the thermal signature cannot produce a variation larger than 2. That means that the thermal signature of cracks is always merged with a large increase of the signal. This is a strong inconvenient since the black body effect can be also observed on any kind of surface defect associated with local variations of $\alpha$ or/and $\varepsilon$, e.g. when scratches pass under the spots of the photothermal camera. To recover the pure thermal signature, a special procedure is necessary [8-10]. This procedure can be separated in two steps: 1 - the recording of two scannings, one from left to right and the other from right to left. Depending on the surface roughness, different offsets between the spots and modifications of the pump distribution may be necessary for the scannings. 2- a subtraction of the recorded signals removes a large part of the optical effects whereas the thermal signature of the cracks is nearly doubled. Furthermore, when the roughness is important or when deep scratches are present, a weighted combination of back and forth signals may be necessary.

In the industrial photothermal camera developed by Framatome, the heat source used to produce the temperature increase is a $100 \mathrm{~W}$ CW YAG laser. In order to improve the productivity, the shape of the spot on the inspected surface is an ellipse of $15 \times 0.1 \mathrm{~mm}^{2}$. The infrared sensor is a short wavelength MCT focal plane array, with $128 \times 128$ pixels working at $200 \mathrm{~Hz}$. The working distance is in the range $900-700 \mathrm{~mm}$. The scanning speed can reach $10 \mathrm{~cm} / \mathrm{s}$ which leads to a maximum velocity of inspection $5 \mathrm{~cm}^{2} / \mathrm{s}$. The performances are illustrated in figs. 2 and 3.

In fig. 2 the object is a $20 \mathrm{~cm}$ plasma coated steel disc in which cracks of openings from 1 to $15 \mu \mathrm{m}$ are present. A photography showing the results of penetrants inspection is also provided: five openings cracks are detected but the external diameter of the coating also produces a signal. The five opening cracks are detected by the photothermal camera with the typical thermal signature. The external diameters present bipolar signatures too but they are inverted with respect to the ones of the cracks. From this result it can be deduced that they should be related to the topography and not to defects as the result by penetrants could let think. Finally, thanks to the back and forth scanning procedure, no false alarm is produced by the roughness which is almost invisible in the resulting photothermal image. This image was obtained in about two minutes.

Several measurements on calibrated cracks have shown that the limit of detection is probably better that the one of the penetrants since openings smaller than $1 \mu \mathrm{m}$ were clearly detected.

To evaluate the spatial resolution, six notches have been prepared in a piece of steel with separations of $4 \mathrm{~mm}$ and $1 \mathrm{~mm}$ (fig. 3). The thermal signatures are clearly separated even at $1 \mathrm{~mm}$.

\section{Interpretation of the raw signals induced by thermal and optical defects}

A good knowledge of the signal build-up over cracks and over those defects that only perturbate the control process (surface roughness, rust, ...) is necessary. It helps for the optimization of the detection procedure leading to an increase of the thermal contrast and a further improvement of the discrimination between cracks and non-dangerous defects.

Due to the thermal diffusion and to the finite size of both the heating spot and the detection spot, a point-like or a line-like defect appears spread over a finite width. A good knowledge of this blurring effect is important to better control the camera performances. The spatial resolution of the flying spot camera is however not well documented in the literature. An analytical model was thus developped to predict the signal contrast appearing over the following photothermal inhomogeneities: a joint emissivity and absorptivity variation on the one hand; and a perpendicular thermal resistance on the other hand [10].

\subsection{Detection of open cracks}

Let us assume that the material contains an open crack. This defect is modelled by an infinite resistance perpendicular to the surface. It thus separates in two symmetric parts the 
half space representing the inspected material. For sake of simplicity, we assume that the crack is perpendicular to the scanning direction. The temperature field induced by the moving heat source in the vicinity of the defect is well known [4]. It is assumed that the detector aims at some distance off the heating spot, along its path. This offset $x_{d}$ can be either positive or negative (the detector respectively aims ahead or behind the heating spot). The integration of the signal according to the spatial responsivity of the IR detector reveals that $r_{s}$ and $r_{d l}$ (the spot radius of respectively the heating source and the detector image - both assumed gaussian) no longer intervene through solely $r_{e}=\sqrt{r_{s}^{2}+r_{d}^{2}}$, the equivalent focusing spot radius of the camera which alone charaterizes the nominal signal level [10]. We thus chose $r_{s}$ as the normalizing parameter (a Péclet number is then defined as follows: $P e=V r_{d} / a$ where $a$ is the material diffusivity and $V$ the scanning speed).

A typical signal evolution when the IR detector crosses a crack is shown in Fig. 4 (left). One recognizes the previously mentioned bipolar shape. Thermal diffusion and the finite value of the laser and detector spot size make the bipolar signature more or less sharp. The image obtained by raster scanning of the surface therefore appears more or less blurred. It was our objective to evaluate the influence of the camera parameters on this aspect. For the purpose of quantification we introduced the peak-to-valley distance $\delta$ as the "resolution limit » of the camera with respect to cracks.

From Fig. 4 (right) we can see that in the case of a broad detection $\left(r_{d}>3 r_{s}\right)$ the « resolution limit " is about $r_{d} / 2$ (it doesn't depend on the scanning speed). On the other hand, in the case of a narrow detection, $\delta$ depends both on speed and on the heating spot size. In particular, if the Péclet number is fixed to $1, \delta$ is roughly given by $\sqrt{r_{s} r_{d}}$. The benefit of the $r_{d}$ reduction for improved image sharpness becomes thus progressively lower.

On the other hand, as was shown in [10], an increase of $r_{d} / r_{s}$ over 1 leads to a rapid decrease of the thermal contrast induced by a crack. Alternatively, for a given spatial resolution, i.e. for a given value of $r_{d}$, it was recommended to focus the laser beam such that $r_{s}$ is between $0.1 r_{d}$ and $r_{d}$ and to scan with such a speed that $V r_{d} d a$ is below about 1 .

\subsection{Optical variations imaging}

Scratches and rust present local absorptivity and emissivity variations which often modify the nominal temperature field and then the recorded signal. Let us assume that the optical defect is a very narrow strip of width $d x_{0}\left(d x_{0}<<r_{s}\right)$ perpendicular to the heating beam direction. Over this strip the absorptivity and the emissivity change by $\Delta \alpha$ and $\Delta \varepsilon$. The signal variation has three components that are proportional to $\varepsilon \Delta \alpha, \alpha \Delta \varepsilon$ and $\Delta \alpha \Delta \varepsilon[10]$.

An example of this signal variation profile is given in Fig. 5 (left). As $\Delta \alpha$ and $\Delta \varepsilon$ were both taken to be positive, the signal presents a "bump ». Due to both thermal diffusion and scanning processes, this "bump » is not perfectly symmetrical. Although the optical defect was assumed to be very thin, the signal variation is relatively extended. From its half-width $\delta$ we defined the « resolution limit » of the camera with respect to optical defects.

In Fig. 5 (right) we plotted the resolution limit for the case of equal absorptivity and emissivity variations. Other results were published elsewhere [10]. In the case of narrow detection $\left(r_{d}<r_{s}\right)$ the defect essentially acts through its emissivity variation. The resolution limit is then roughly equal to $r_{d}$, whatever the scanning speed could be. On the other hand, in the case of a relatively broad detection $\left(r_{d} \gg r_{s}\right)$, the resolution limit gets near $f(P e) r_{s}$, where $f(P e)$ varies between 2.3 and 3 for Pe between 0.1 and 10 .

\section{Conclusion}

The detection of perpendicular cracks, impossible by flash thermography with uniform heating, is achieved by the photothermal camera. Today available procedure makes the system almost insensitive to the surface defects. 
The theoretical analysis has shown that the degree of sharpness of the images produced by the flying spot camera strongly depends on the nature of the " defects $*$ that are present on the heating beam path. Optical defects look sharper than cracks. Only when the heating spot and the detection spot have similar size can we expect that the "resolution limit » will be of the same order for both type of defects. The obtained results will help for a further refinement of the normalization procedure previously described which is based on back and forth scanning.

The prototype that was built already indicates that the photothermal camera could replace the penetrants in the next future when they present difficult or dangerous application.

\section{REFERENCES}

[1] KUBIAK (E. J.) - Infrared detection of fatigue cracks and other near-surface defects, Applied Optics, vol. 7(9), 1968, p. 1743-1747.

[2] KAUFMAN (I.), CHANG (P. T.), HSU (H. S.), HUANG (W. Y.), SHYONG (D. Y.) - Photothermal radiometric detection and imaging of surface cracks. J. Nondestructive Evaluation, vol. 3(2), 1987, p. 87-100.

[3] WANG (Y. Q.), KUO (P. K.), FAVRO (L. D.), THOMAS (R. L.) - Flying laser spot thermal wave IR imaging of horizontal and vertical cracks. Review of Progress in Quantitative Nondestructive Evaluation. Thompson (D.O.) and Chimenti (D.E.) eds., Plenum Press, New York, vol. 9, 1990, p. 511-516.

[4] GRUSS (C.), BALAGEAS (D.) - Theoretical and experimental applications of the flying spot camera. Proc. of Quantitative Infrared Thermography, QIRT 92, (Eurotherm Seminar 27), Balageas (D.) et al. eds., Ed. Eur. Thermique et Industrie, Paris, 1992, p. 19-24.

[5] BODNAR (J.-L.), EGEE (M.), MENU (C.), BESNARD (R.), LE BLANC (A.), PIGEON (M.), SELLIER (J.-Y.) - Cracks detection by a moving photothermal probe. 8th Int. Topical Meeting on Photoacoustic and Photothermal Phenomena, Fournier (D.) and Roger (J.P.) eds., Les Editions de la Physique, 1994, p. 591-594.

[6] VARIS (J.), OKSANEN (M.), RANTALA (J.), LUUKKALA (M.) - Observations on image formation in the line scanning thermal imaging method. Review of Progress in Quantitative Nondestructive Evaluation, ibid., vol. 14, 1995, p. 447-452.

[7] VARIS (J.), LEHTINIEMI (R.) - A thermal nondestructive evaluation system for detecting vertical cracks in unidirectional carbon fiber composites. Rev. Sci. Instrum. vol. 68(7), 1997, p. 2818-2821.

[8] LEGRANDJACQUES (L.), KRAPEZ (J.-C.), LEPOUTRE (F.), BALAGEAS (D.) - Détection de fissuration transverse par une caméra photothermique. Congrès SFT97, Toulouse, 2022 mai 1997, Elsevier, Paris, 1998 [in French].

[9] LEGRANDJACQUES (L.), KRAPEZ (J.-C.), LEPOUTRE (F.), BALAGEAS (D.) - Nothing but the cracks: a new kind of photothermal camera. 7th ECNDT, Copenhaguen, 26-29 may 1998.

[10] KRAPEZ (J.-C.) - Résolution spatiale de la caméra à source volante. accepted for publ. in Int. J. Thermal Sci. [in French]. 


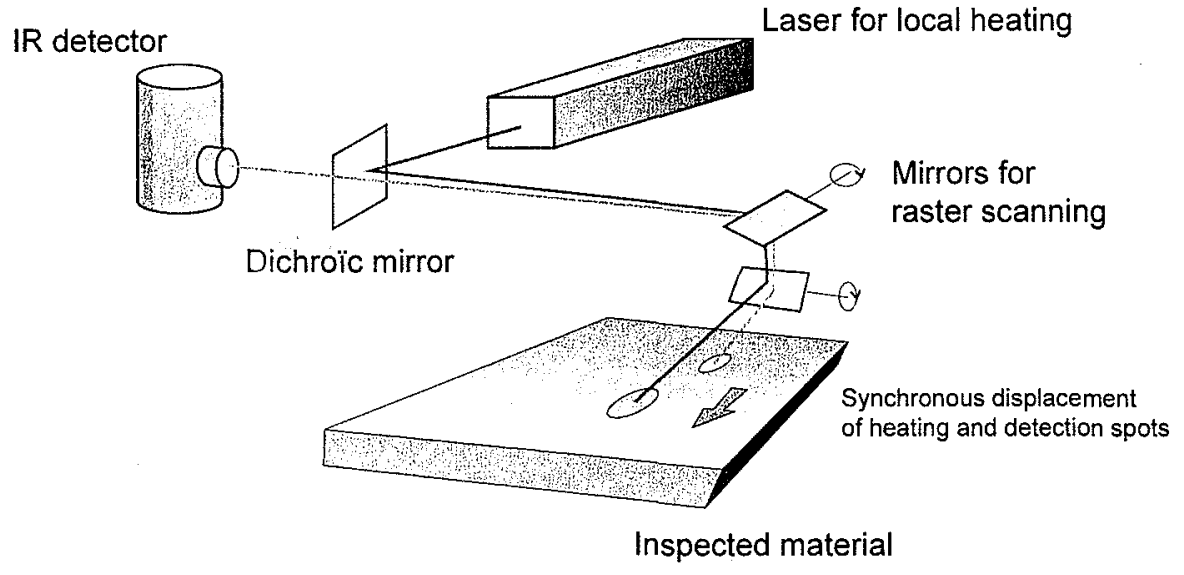

Fig. 1. Principle of the photothermal or flying spot camera

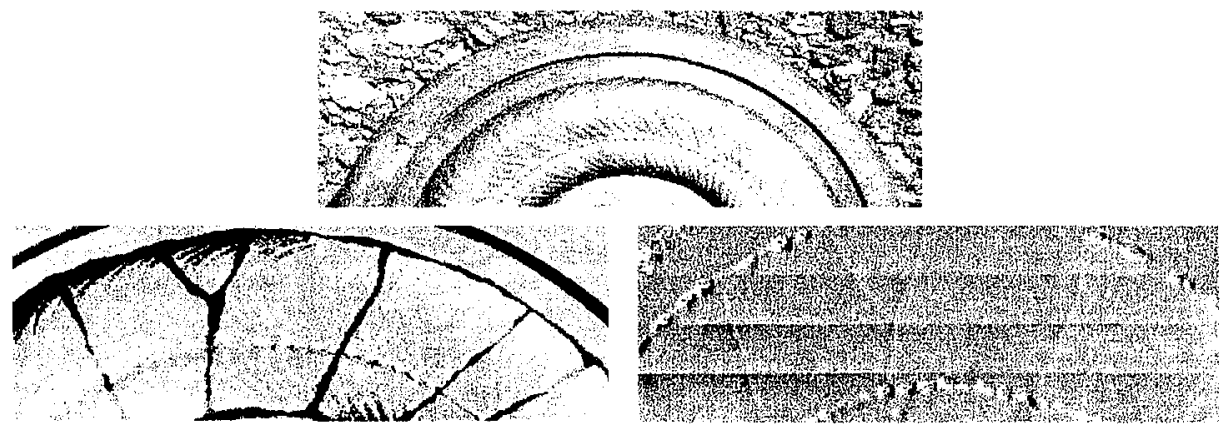

Fig. 2. Partial view of the inspected disc (top), penetrant testing result (bottom left) and photothermal image of the sample (bottom right). Inspection speed is about $1 \mathrm{~m}^{2}$ per hour
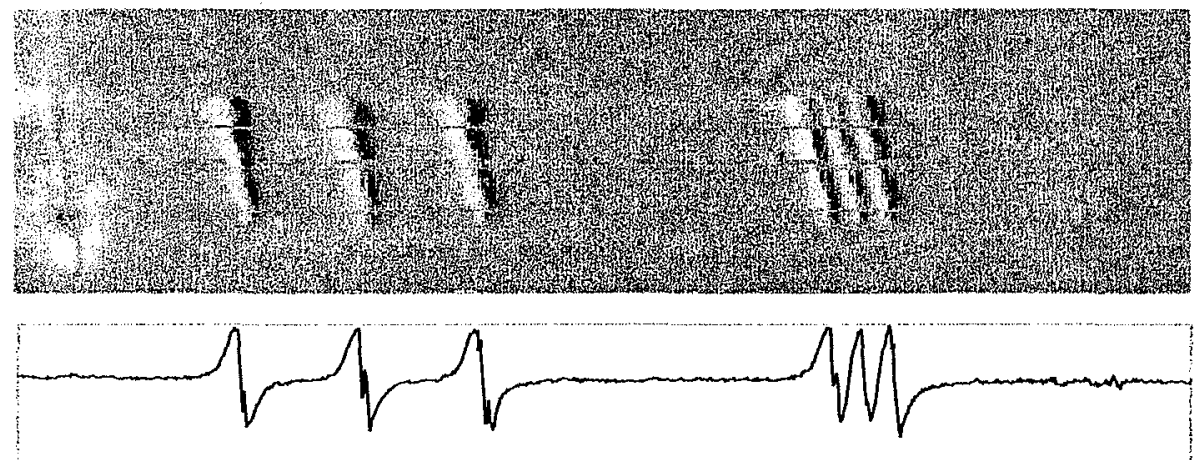

Fig. 3. Spatial resolution. The inter-notch steps are equal to $4 \mathrm{~mm}$ (left) and $1 \mathrm{~mm}$ (right) 
http://dx.doi.org/10.21611/qirt.1998.048
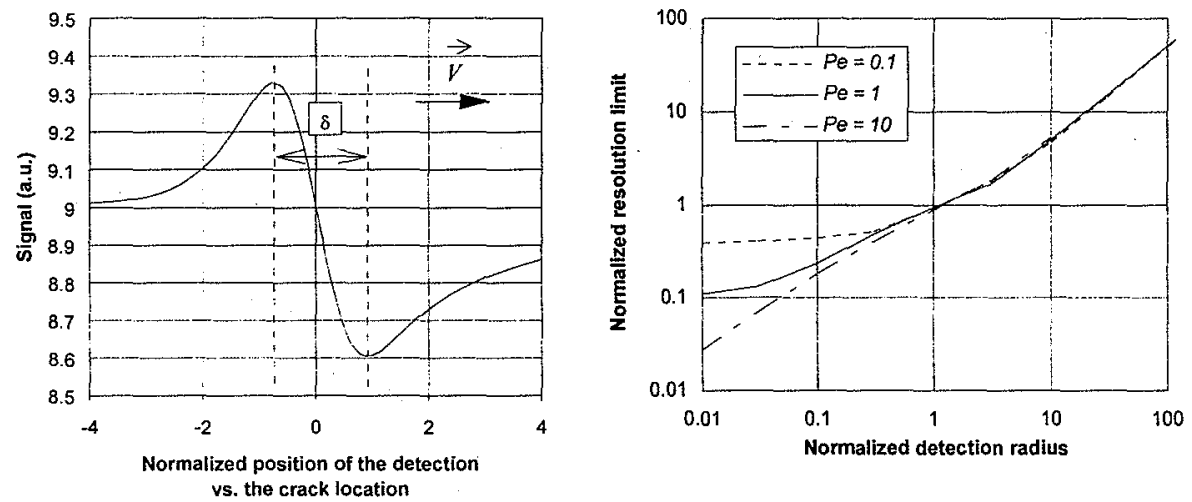

Fig. 4. Left: theoretical signal obtained when crossing a crack $\left(r_{d}=3 r_{s}, x_{d}=0, P e=1\right)$, Right: normalized resolution limit $\delta / r_{s}$ when the imaged defect is a perpendicular crack (offset kept at 0 )
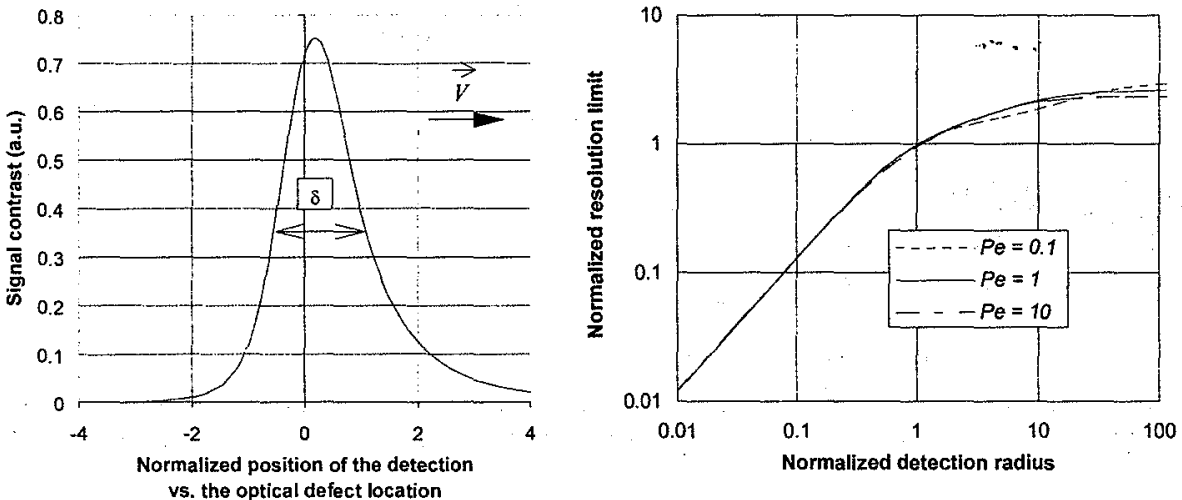

Fig. 5. Left: theoretical signal obtained when crossing an optical defect

$\left(r_{d}=3 r_{s}, x_{d}=0, P e=1\right.$, absorptivity and emissivity both jump from 0.2 to 1) Right: normalized resolution limit $\delta / r_{s}$ when the imaged defect is purely optical (offset $=0$ ) 